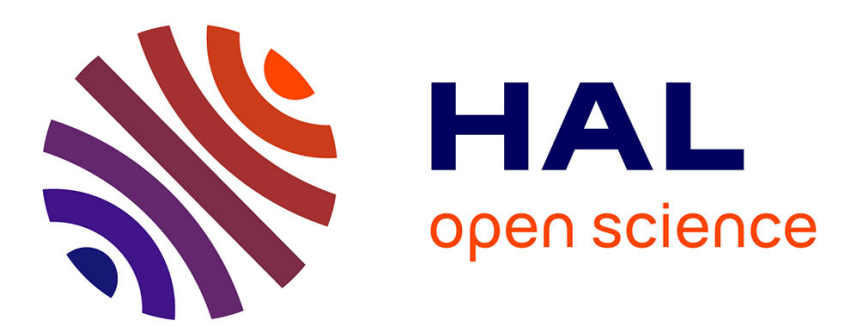

\title{
Degeneracy of the crystal states and departure from the rigid band model for a strongly localized impurity
}

\author{
S. Olszewski
}

\section{To cite this version:}

S. Olszewski. Degeneracy of the crystal states and departure from the rigid band model for a strongly localized impurity. Journal de Physique Lettres, 1985, 46 (15), pp.703-709. 10.1051/jphyslet:019850046015070300 . jpa-00232583

\section{HAL Id: jpa-00232583 https://hal.science/jpa-00232583}

Submitted on 1 Jan 1985

HAL is a multi-disciplinary open access archive for the deposit and dissemination of scientific research documents, whether they are published or not. The documents may come from teaching and research institutions in France or abroad, or from public or private research centers.
L'archive ouverte pluridisciplinaire HAL, est destinée au dépôt et à la diffusion de documents scientifiques de niveau recherche, publiés ou non, émanant des établissements d'enseignement et de recherche français ou étrangers, des laboratoires publics ou privés. 
Classification

Physics Abstracts

71.55

\title{
Degeneracy of the crystal states and departure from the rigid band model for a strongly localized impurity
}

\author{
S. Olszewski \\ Institute of Physical Chemistry, Polish Academy of Sciences. Warsaw, Poland
}

(Reçu le 6 juin 1985, accepté le 11 juin 1985)

\begin{abstract}
Résumé. - Un calcul de perturbation standard est effectué pour un ensemble de $q$ états dégénérés perturbés par une impureté fortement localisée. L'approximation des électrons libres et celle des combinaisons linéaires d'orbitales atomiques sont considérées. Le calcul montre qu'un des états est fortement perturbé en énergie, tandis que les $q-1$ autres ne le sont pas. L'effet est indépendant de la taille du potentiel perturbateur si le calcul de perturbation est applicable. L'état déplacé n'est pas localisé mais est une combinaison d'états de bande non perturbés. Pour un cristal de forme sphérique, la distribution de charge de l'état déplacé décroît de façon oscillante avec la distance à l'impureté.
\end{abstract}

\begin{abstract}
A standard perturbation calculation is performed for a set of $q$ degenerate states whose perturbation is a strongly localized impurity. The free-electron approximation and the LCAO approximation for the electron states are considered. The calculation which seems not to have been done before shows that there is precisely no change in the energy for $q-1$ states but a strong shift of energy for one state. This effect is fully independent of the size of the perturbation potential providing the perturbation calculation is applicable. At the same time the wave function corresponding to the shifted state is not a damped state but a simple superposition of the unperturbed band states. For a crystal having a spherical shape it can be shown that the charge distribution of the shifted state has an oscillatory decrease with the distance from the position of the impurity.
\end{abstract}

\section{Introduction.}

Friedel and Blandin [1-3] were the first who discussed the idea of the virtual bound state in a metal; cf. also reference [4]. This state comes out from the degeneracy between a localized impurity state and an extended band state. The effect of the formation of the virtual bound state is a local increase of the density of states plotted within the band versus energy accompanied by an accumulation of electron charge about the impurity. The centre of the accumulated charge is at the impurity position. Anderson [5] and Wolff [6] gave an extended explanation of this effect. In particular, in Wolff's explanation the virtual bound state corresponded to a rather sharp maximum of the scattering amplitude encountered by the electrons of the matrix metal on the impurity position. All these models go beyond the so-called rigid-band approximation for the metal electrons. This approximation is based on the first-order perturbation calculation in which the wave functions of the metal matrix are not modified but only the energy perturbation of the metal states is considered. Mathematically, the sense of the rigid-band approximation is to neglect 
the matrix elements of the perturbation potential between different Bloch states but consider solely the matrix elements between the same states, viz.

$$
V_{\mathbf{k k}}=\int \psi_{\mathbf{k}}^{*}(\mathbf{r}) V(\mathbf{r}) \psi_{\mathbf{k}}(\mathbf{r}) \mathrm{d} \mathbf{r}
$$

where $\psi_{\mathbf{k}}(\mathbf{r})$ is the Bloch wave function of the matrix metal and $V(\mathbf{r})$ is the perturbation potential. In this paper we show that the effects similar to those given by the virtual bound states can also be attained on the basis of a standard first-order perturbation calculation providing the degeneracy of the Bloch states - neglected in the rigid-band model - is now taken into account. So, in the present approach, we do an inclusion of

$$
V_{\mathbf{k}^{\prime} \mathbf{k}}=\int \psi_{\mathbf{k}^{\prime}}(\mathbf{r}) V(\mathbf{r}) \psi_{\mathbf{k}}(\mathbf{r}) \mathrm{d} \mathbf{r}
$$

where $\mathbf{k}^{\prime} \neq \mathbf{k}$, together with (1) and perform the diagonalization process dictated by the firstorder perturbation calculation. At the same time a strong localization of the impurity potential is assumed. As the first step let us consider a situation when the band states can be approximated by the free-electron states.

\section{The perturbation model for the free-electron states.}

We represent the metal electrons by a very large number of the plane wave functions

$$
\Omega^{-1 / 2} \mathrm{e}^{i \mathbf{k}^{\prime} \mathbf{r}}, \Omega^{-1 / 2} \mathrm{e}^{i \mathbf{k}^{\prime \prime} \mathrm{r}}, \Omega^{-1 / 2} \mathrm{e}^{i \mathbf{k}^{\prime \prime} \mathbf{r}} \ldots
$$

for which

$$
\left|\mathbf{k}^{\prime}\right|=\left|\mathbf{k}^{\prime \prime}\right|=\left|\mathbf{k}^{\prime \prime \prime}\right|=\cdots=k,
$$

thus the functions form a degenerate set for the free-electron Hamiltonian, $\Omega$ is the metal volume. The functions (3) are orthogonal and normalized in $\Omega$. They can be represented as linear combinations of the other degenerate and orthogonal set of functions, viz.

$$
\psi_{l m}=N_{l m} Y_{l m}(\theta, \zeta) j_{l}(k r)
$$

$N_{l m}$ is a normalization coefficient, $Y_{l m}(\theta, \zeta)$ is a spherical harmonic, and $j_{l}(k r)$ is a spherical Bessel function. It is convenient to normalize functions (5) in a sphere of radius $R_{\mathrm{d}}$, so we have

$$
N_{l m} \cong\left(\frac{k^{2}}{2 \pi R_{\mathrm{d}}}\right)^{1 / 2}
$$

for any not too large $l$ and $m$ providing $R_{\mathrm{d}}$ is a very large distance and the asymptotic form of $j_{l}(k R)$ at large $k R$ is taken into account.

The free-electron wave functions (5) are all of the same energy $k^{2}$. Assuming the perturbation potential $V(\mathbf{r})$ is given by an impurity which is strongly localized at the position $\mathbf{r}=\mathbf{0}$, viz.

$$
V(\mathbf{r})=V_{0} \delta(\mathbf{r}-\mathbf{0}),
$$

where $\delta$ is the delta-function, we have to perform a standard first-order perturbation calculation for the degenerate states. This requires to solve the secular equation 


$$
\left|\begin{array}{l}
\left\langle\psi_{1}|V| \psi_{1}\right\rangle-\Delta E,\left\langle\psi_{1}|V| \psi_{2}\right\rangle,\left\langle\psi_{1}|V| \psi_{3}\right\rangle, \ldots \\
\left\langle\psi_{2}|V| \psi_{1}\right\rangle,\left\langle\psi_{2}|V| \psi_{2}\right\rangle-\Delta E,\left\langle\psi_{2}|V| \psi_{3}\right\rangle, \ldots \\
\left\langle\psi_{3}|V| \psi_{1}\right\rangle,\left\langle\psi_{3}|V| \psi_{2}\right\rangle,\left\langle\psi_{3}|V| \psi_{3}\right\rangle-\Delta E, \ldots \\
\ldots \ldots \ldots \ldots \ldots \ldots \ldots \ldots \ldots \ldots \ldots \ldots \ldots \ldots \ldots \ldots \ldots \ldots \ldots \ldots
\end{array}\right|=0
$$

where

$$
\Delta E=E-k^{2}
$$

and $E$ is the energy of the perturbed state. Different pairs of the indices $l$ and $m$ are abbreviated by $1,2,3 \ldots$ For the potential (7) equation (8) becomes extremely simple because all the elements of the secular determinant (8) vanish except for

$$
\left\langle\psi_{1}|V| \psi_{1}\right\rangle=V_{0}\left\langle\psi_{00}|\delta(\mathbf{r}-\mathbf{0})| \psi_{00}\right\rangle=V_{0} N_{00}^{2} j_{0}^{2}(0)=V_{0} \frac{k^{2}}{2 \pi R_{\mathrm{d}}} .
$$

This is so because of the well-known property

$$
j_{l}(0)=\delta_{l 0}
$$

where $\delta$ is the Kronecker symbol. We obtain

$$
\Delta E=V_{0} \Delta E=V_{0} \frac{k^{2}}{2 \pi R_{\mathrm{d}}},
$$

hence only one wave function (5), namely that having $l=m=0$, is modified by the perturbation (7). We can multiply (12) by (i) $R_{\mathrm{d}} / \pi$ - which is the density of the free-electron states [7] per unit of $k$ - and (ii) the $\mathbf{k}$-space volume factor $(2 \pi)^{3}$. This gives

$$
\Delta E \frac{R_{\mathrm{d}}}{\pi}(2 \pi)^{3}=V_{0} 4 \pi k^{2}
$$

We obtain the shift of energy of one state (5) proportional to the perturbation strength $V_{0}$ and the degeneracy $4 \pi k^{2}$ of the states (5). The remainder of functions (5) is left unmodified, so is a given unperturbed energy

$$
E_{0}=k^{2}
$$

is considered - the perturbation produces the diminution of the electron charge at $E_{0}$ and the same increase of charge at $E_{0}+\Delta E$. This change of charge is equal to

$$
\rho(\mathbf{r})=N_{00}^{2} j_{0}^{2}(k r)
$$

and represents the well-known Friedel oscillations [3] about position $\mathbf{r}=\mathbf{0}$ decreasing strongly with the distance $|\mathbf{v}|$.

A completely different situation is encountered when the rigid-band model is applied to the wave functions (3). In this case the energy (14) of any wave function is submitted to the change

$$
\Omega^{-1}\left\langle\mathrm{e}^{i \mathbf{k}^{\prime} \mathbf{r}}|V| \mathrm{e}^{i \mathbf{k}^{\prime} \mathbf{r}}\right\rangle=\Omega^{-1}\left\langle\mathrm{e}^{i \mathbf{k}^{\prime \prime} \mathbf{r}}|V| \mathrm{e}^{i \mathbf{k}^{\prime \prime} \mathbf{r}}\right\rangle=\Omega^{-1}\left\langle\mathrm{e}^{i \mathbf{k}^{\prime \prime \prime} \mathbf{r}}|V| \mathrm{e}^{i \mathbf{k}^{\prime \prime \prime} \mathbf{r}}\right\rangle=\cdots=\Omega^{-1} V_{0},
$$

thus the whole electron density given by the wave functions (3) is shifted by the energy amount $\Omega^{-1} V_{0}$; equation (16). To get an agreement of the impurity effect upon the plane waves with the same effect obtained for functions (5) the degeneracy of functions (3) has to be taken into account in the course of the perturbation calculation. This calculation is done below for the general LCAO states and the result for the plane waves can be obtained as a special case. 


\section{The perturbation model for the LCAO states.}

The wave function represented in the LCAO approximation is in general

$$
\psi^{v}=\sum_{\mathbf{R}_{i}} A^{v}\left(\mathbf{R}_{i}\right) \zeta\left(\mathbf{r}-\mathbf{R}_{i}\right)
$$

where $A^{v}\left(\mathbf{R}_{i}\right)$ is the coefficient multiplying the atomic orbital $\zeta\left(\mathbf{r}-\mathbf{R}_{i}\right)$ of the matrix metal localized about the lattice site $\mathbf{R}_{i}$, index $v$ labels different electron states; in the Bloch theory $v$ is a $\mathbf{k}$-vector. For the sake of simplicity we assume only one kind of $\zeta\left(\mathbf{r}-\mathbf{R}_{i}\right)$ in the metal. Since the impurity potential is localized at site 0 , the matrix element between the wave function $v=\alpha$ and the wave function $\delta=\beta$ is

$$
V(\mathbf{0}) A^{\alpha}(\mathbf{0}) A^{\beta}(\mathbf{0}),
$$

providing that $A^{\alpha}(\mathbf{0})$ and $A^{\beta}(0)$ are real quantities;

$$
V(\mathbf{0})=\int \zeta^{*}(\mathbf{r}-\mathbf{0}) V(\mathbf{r}) \zeta(\mathbf{r}-\mathbf{0}) \mathrm{d} \mathbf{r} .
$$

Let us note that (18) is the square root of the product of two diagonal matrix elements, viz.

and

$$
a=V(\mathbf{0}) A^{\alpha}(\mathbf{0}) A^{\alpha}(\mathbf{0})
$$

$$
b=V(\mathbf{0}) A^{\beta}(\mathbf{0}) A^{\beta}(\mathbf{0}),
$$

so we may put

$$
(a b)^{1 / 2}=V A^{\alpha}(\mathbf{0}) A^{\beta}(\mathbf{0}) .
$$

If the two wave functions, $v=\alpha$ and $v=\beta$, are degenerate having energy $E_{0}$, the perturbation splits this energy into two levels $E=E_{(1)}$ and $E=E_{(2)}$ according to the secular equation

$$
\left|\begin{array}{cc}
a-\Delta E, & (a b)^{1 / 2} \\
(a b)^{1 / 2}, & b-\Delta E
\end{array}\right|=0
$$

in which

$$
\Delta E_{(i)}=E_{(i)}-E_{0} .
$$

The solution of (23) gives

$$
\begin{aligned}
& \Delta E_{(1)}=0 \\
& \Delta E_{(2)}=a+b .
\end{aligned}
$$

The resulted wave functions obtaindd for any of the two $E$ calculated from (25) and (26), respectively, are certain linear combinations of $\psi^{\alpha}$ and $\psi^{\beta}$; to be correct approximations of the wave functions these two combinations have to be orthogonal.

Taking into account a set of $q$ degenerate states

$$
v=\alpha, \beta, \gamma, \delta, \varepsilon \ldots
$$

and putting

$$
\begin{aligned}
& c=V(\mathbf{0}) A^{\gamma}(\mathbf{0}) A^{\gamma}(\mathbf{0}), \\
& d=V(\mathbf{0}) A^{\delta}(\mathbf{0}) A^{\delta}(\mathbf{0}), \\
& e=V(\mathbf{0}) A^{\varepsilon}(\mathbf{0}) A^{\varepsilon}(\mathbf{0}),
\end{aligned}
$$


we obtain - together with the expressions (20) and (21) - the secular equation of $q$ th order :

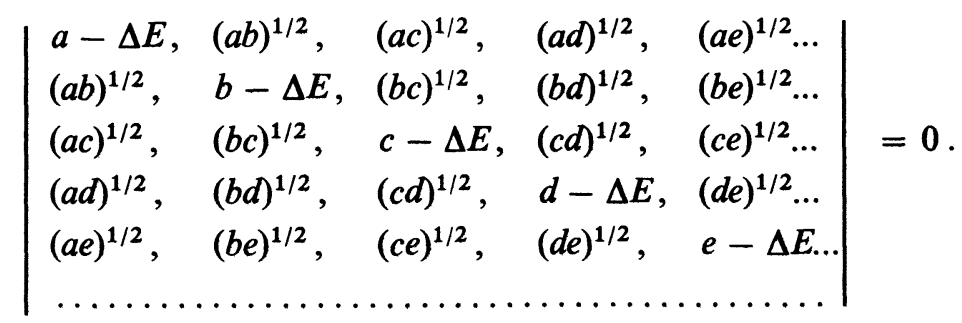

A characteristic point is that $q-1$ solutions of this equation, say those labelled by the indices from 1 to $q-1$, give

$$
\Delta E_{(1)}=\Delta E_{(2)}=\Delta E_{(3)}=\cdots=\Delta E_{(q-1)}=0
$$

whereas one of the solutions, that labelled by the index $q$, gives

$$
\Delta E_{(q)}=a+b+c+d+e+\cdots .
$$

This result has been obtained by inspection beginning from the $2 \times 2$ secular equation (23) and increasing gradually the size of the secular determinant (8). The wave functions corresponding to energies (32) and (33) are again linear combinations of the states listed in (27). These combinations can be made mutually orthogonal.

We now get a net distinction between the rigid-band model and the present approach. In the rigid-band model the energy of any state is shifted individually by $a, b, c, \ldots$; see equations (20), (21) and (28)-(30). In the present case the shift of one state is given by the sum of the individual shifts calculated in the rigid-band model (Eq. (33)), whereas the remainder of the shifts are zero (Eq. (32)). In each case the sum of the shifts calculated for all states is the same, since the trace of the Hamiltonian calculated over two sets of the wave functions which differ by a unitary transformation has to remain unchanged.

In case $\alpha, \beta, \gamma \ldots$ are the Bloch states, we have

$$
A^{\alpha}(\mathbf{0})=A^{\beta}(\mathbf{0})=A^{\gamma}(\mathbf{0})=\cdots=N^{-1 / 2}
$$

since $N^{-1 / 2}$ is the normalization coefficient of Bloch's LCAO state, so

$$
a=b=c=\cdots=V(0) N^{-1} ;
$$

$N$ is the number of atoms in the crystal, providing the atomic orbitals centred on different lattice sites are orthogonal functions. With the aid of (35) expression (33) simplifies into

$$
\Delta E_{(q)}=q V(0) N^{-1},
$$

where $q$ represents the degeneracy of the unperturbed Bloch states, thus the unperturbed Bloch energies

$$
E_{\alpha}=E_{\beta}=E_{\gamma}=\cdots=E_{0}
$$

listed on the left of $E_{0}$ are $q$ in number.

A much similar situation is for the plane waves given in (3). Here the matrix elements satisfy the relation

$$
a=b=c=\cdots=V_{0} \Omega^{-1}
$$


and $E_{0}$ is $k^{2}$; the symbol $V_{0}$ is defined in (7). We obtain

$$
\Delta E_{(q)}=q V_{0} \Omega^{-1}
$$

and the remainder of $\Delta E_{(i)}$ - where $i=1,2 \ldots, q-1-$ satisfies equation (32). The boundary conditions should not influence the solution within the metal. Therefore we may expect the density distribution corresponding to the electron state shifted by (39), as well as that of the state shifted by (36), oscillate in the way shown for the density (15).

\section{Perturbation calculation for the standing LCAO wave functions.}

Here the LCAO wave function is the basis function of an irreducible representation of the crystal point group and the coefficient function has the form of a standing wave. Assuming any wave function is based on the atomic orbitals of type $s$ and the crystal symmetry is that of a cube only, the wave functions belonging to the total-symmetry representation $\Gamma_{1}$ are modified by the impurity potential $V(\mathbf{v})$ located about the atomic position $\mathbf{R}=0$. Any unperturbed wave function belonging to $\Gamma_{1}$ has its coefficient function

$$
A^{\Gamma_{1}, \lambda}(k, \mathbf{R})
$$

of the same symmetry. The index $\lambda$ takes into account the degeneracy of a given unperturbed energy $E_{0}$, so any $E_{0}$ is connected with a set of the coefficient functions

$$
A^{\Gamma_{1}, 1}\left(k^{\prime}, \mathbf{R}\right), \quad A^{\Gamma_{1,2}}\left(k^{\prime \prime}, \mathbf{R}\right), \quad A^{\Gamma_{1}, 3}\left(k^{\prime \prime \prime}, \mathbf{R}\right) \ldots
$$

which satisfy the Wannier-Slater eigenequation for a given crystal lattice $[9,10]$. In the case of a crystal with spherical shape it is convenient to represent any of these coefficient functions as a linear combination of the products calculated between the spherical Bessel functions $j_{l}$ of a given $l$ and the cubic harmonic $(K H)_{l, t}^{\Gamma_{1}}$ having the same $l$ and belonging to $\Gamma_{1}$. This gives

$$
A^{\Gamma_{1}, \lambda}(k, \mathbf{R})=\sum_{l, t} c_{l, t}^{\Gamma_{1}, \lambda}(k)(K H)_{l, t}^{\Gamma_{1}}(\theta, \varphi) j_{l}(k R)
$$

where the coefficients $c_{l, t}^{\Gamma, \lambda}(k)$ are obtained from the solution of the Wannier-Slater equation. The scalar parameters $k^{\prime}, k^{\prime \prime}, k^{\prime \prime \prime} \ldots$ entering (41) may be different for any coefficient function corresponding to a given $E_{0}$.

The rigid-band model gives the energy change

$$
\begin{aligned}
& \Delta E_{1}^{\mathrm{rig}}=V(\mathbf{0})\left[A^{\Gamma_{1}, 1}\left(k^{\prime}, \mathbf{0}\right)\right]^{2}, \\
& \Delta E_{2}^{\mathrm{rig}}=V(\mathbf{0})\left[A^{\Gamma_{1}, 2}\left(k^{\prime \prime}, \mathbf{0}\right)\right]^{2}, \\
& \Delta E_{3}^{\mathrm{rig}}=V(\mathbf{0})\left[A^{\Gamma_{1}, 3}\left(k^{\prime \prime \prime}, 0\right)\right]^{2},
\end{aligned}
$$

respectively for any function given in (41). The matrix element $V(0)$ (Eq. (19)) is calculated between the atomic orbitals of kind s. The number $\lambda_{\max }$ of the functions (41) which have a given $E_{0}$ is the degeneracy $q$ of $E_{0}$. The perturbation calculation presented in section 3 and applied to the functions (41) gives the following corrections for $E_{0}$ :

and

$$
\Delta E_{(1)}^{\mathrm{deg}}=\Delta E_{(2)}^{\mathrm{deg}}=\Delta E_{(3)}^{\mathrm{deg}}=\cdots=\Delta E_{(q-1)}^{\mathrm{deg}}=0
$$

$$
\Delta E_{(q)}^{\mathrm{deg}}=\Delta E_{1}^{\mathrm{rig}}+\Delta E_{2}^{\mathrm{rig}}+\cdots+\Delta E_{q}^{\mathrm{rig}} .
$$


Basing ourselves on the result obtained for the free-electron wave functions extended within a sphere, we may expect the LCAO wave functions corresponding to (43) to be practically unmodified by the perturbation, whereas the wave function corresponding to (44) gives the oscillating change of the electron density accumulated about the impurity position in $\mathbf{0}$. In fact, the perturbed charge about the position $\mathbf{0}$ is given solely by the wave functions which built up the electron density at $\mathbf{0}$, since only these wave functions (symmetry $\Gamma_{1}$ ) can combine into the perturbed state (44). At the lattice sites which are far from 0 the contribution to electron density given by the wave functions of symmetry $\Gamma_{1}$ is in average only about $1 / 48$ of the total electron density calculated at these sites [11]. Since any $\left[A^{\Gamma_{1}, \lambda}(k, \mathbf{R})\right]^{2}$ oscillates in space decreasing with the distance from 0 [cf. Refs. [9-11] and Eq. (40a)], the same applies to the square of the combination of $A^{\Gamma_{1}, \lambda}(k, \mathbf{R})$ which represents the perturbed state.

\section{Acknowledgments.}

I am profoundly indebted to Professor J. Friedel for his criticism.

\section{References}

[1] Friedel, J., Can. J. Phys. 34 (1956) 1190.

[2] Blandin, A. and Friedel, J., J. Phys. Radium 20 (1959) 160.

[3] Friedel, J., J. Phys. Radium 23 (1962) 692.

[4] DANIEL, E. and Friedel, J., LT 9 (1965) 933.

[5] Anderson, P. W., Phys. Rev. 124 (1961) 41.

[6] Wolff, P. A., Phys. Rev. 124 (1961) 1030.

[7] Friedel, J., Nuovo Cim. Suppl. 7 (1958) 287.

[8] I am grateful to T. KWIATKOWSKI and A. WIERZBICKI for their help in the calculation of the determinants having different sizes.

[9] Olszewski, S., Phys. Rev. B 3 (1971) 4361.

[10] Modrak, P. and Olszewski, S., Phys. Rev. B 14 (1976) 2387.

[11] Bulski, T., OlsZewski, S. and WierZBicki, A., Intern. J. Quant. Chem. 17 (1980) 697. 